\title{
Correction to: Variable decomposition approach applied to multi- objective optimization for minimum powering of commercial ships
}

\author{
Yusuke Tahara $^{1}$ (D) $\cdot$ Yasuo Ichinose $^{1} \cdot$ Azumi Kaneko $^{1} \cdot$ Yoshikazu Kasahara $^{1}$
}

Published online: 18 June 2018

(c) JASNAOE 2018

\section{Correction to: Journal of Marine Science and Technology https://doi.org/10.1007/s00773-018-0551-5}

In the original publication of the article Figs. 14, 26b, e, Eqs. (2), (23), (24), (27), (29) and (30) are incorrectly published. The correct figures and equations are provided in this article.
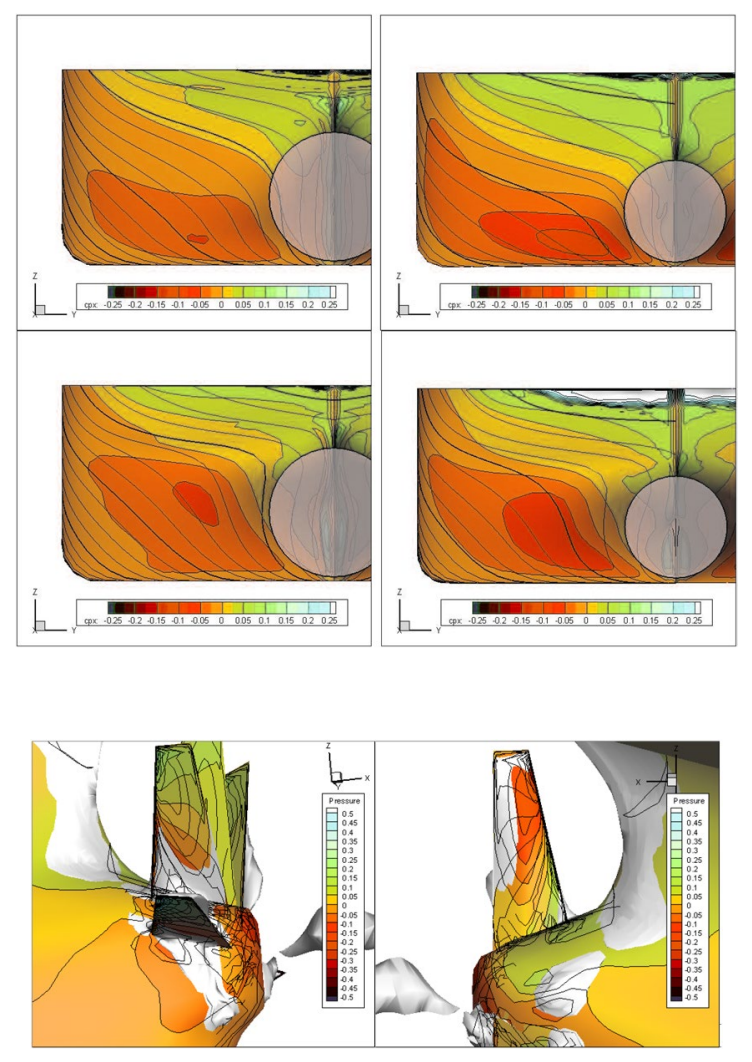

The original article can be found online at https://doi.org/10.1007/ s00773-018-0551-5.

Yusuke Tahara

tahara@nmri.go.jp

1 NMRI, National Maritime Research Institute, Mitaka, Japan

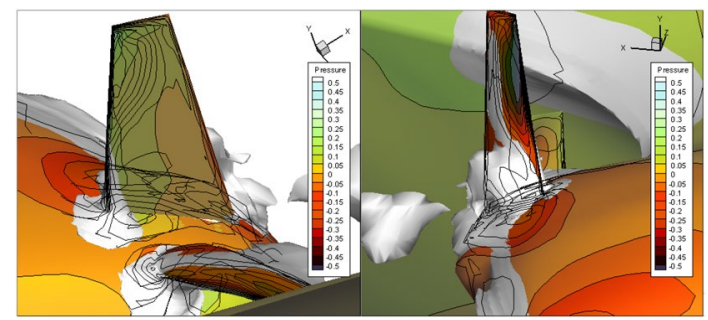

$\frac{\delta \mathrm{DP}}{\mathrm{DP}}=\frac{\delta R}{R}-\frac{\delta \eta_{\mathrm{P}}}{\eta_{\mathrm{P}}}$

$\mathrm{DP} \propto \frac{\rho \pi D^{2}}{16 \alpha_{P} \eta_{R}} V_{\mathrm{a}}^{3} \beta C_{\mathrm{th}}{ }^{r}$

$=\frac{8^{r}\left(\rho \pi D^{2}\right)^{1-r} \beta V_{S}^{3-2 r}}{16 \alpha_{\mathrm{P}} \eta_{R}}\left(1-w_{\mathrm{S}}\right)^{3-2 r} R^{r}(1-t)^{-r}$

$\mathrm{DP} \propto \frac{1}{\eta_{R}}\left(1-w_{\mathrm{S}}\right)^{3-2 r} R^{r}(1-t)^{-r}$

$\frac{1}{r} \frac{\delta \mathrm{DP}}{\mathrm{DP}} \approx \frac{\delta R}{R}-\frac{\delta(1-t)}{(1-t)}+0.439 \frac{\delta\left(1-w_{\mathrm{S}}\right)}{\left(1-w_{\mathrm{S}}\right)}-0.813 \frac{\delta \eta_{R}}{\eta_{R}}$

$$
\begin{aligned}
\frac{1}{r} \frac{\delta \mathrm{DP}}{\mathrm{DP}} & =-\frac{\delta(1-t)}{(1-t)}+\frac{3-2 r}{r} \frac{\delta\left(1-w_{\mathrm{S}}\right)}{\left(1-w_{\mathrm{S}}\right)}-\frac{1}{r} \frac{\delta \eta_{R}}{\eta_{R}} \\
& \approx-\frac{\delta(1-t)}{(1-t)}+0.439 \frac{\delta\left(1-w_{\mathrm{S}}\right)}{\left(1-w_{\mathrm{S}}\right)}-0.813 \frac{\delta \eta_{R}}{\eta_{R}}
\end{aligned}
$$

$$
\frac{1}{r} \frac{\delta \mathrm{DP}}{\mathrm{DP}} \approx-\frac{\delta(1-t)}{(1-t)}+0.439 \frac{\delta\left(1-w_{t}\right)}{\left(1-w_{t}\right)}-0.813 \frac{\delta \eta_{R}}{\eta_{R}}
$$

